\title{
La gestion de la pandémie de COVID-19 au Cameroun : bilan et perspectives
}

\author{
Jacob Atangana-Abéa
}

RÉSUMÉ. À partir de l'exploitation de données secondaires, cet article fait une évaluation de la gestion de la pandémie de COVID-19 par le gouvernement camerounais. Cette évaluation couvre la période de mars 2020, date de début de la pandémie au Cameroun, à la fin du mois d'août 2020. La période en étude est certes courte, mais nous semble suffisante pour tirer les premiers enseignements de la gestion de cette pandémie. Nous soutenons qu'avec la récurrence des catastrophes naturelles et humaines et leurs effets sur la vulnérabilité des populations, le gouvernement camerounais devrait désormais considérer la gestion des catastrophes comme relevant des actes courants de gouvernance du pays et mettre en place des mesures pérennes de soutien aux populations victimes, plutôt que d'agir par à-coups, comme c'est le cas présentement. Car si les crises passent, leurs effets perdurent.

\begin{abstract}
Based on the use of secondary data, this article assesses the management of the COVID-19 pandemic by the Cameroonian government. This assessment spans from March 2020, the beginning of the pandemic in Cameroon, until the end of July 2020. The timeframe under study is certainly short, but appears sufficiently lengthy to draw initial lessons learnt from the management of this pandemic. We maintain that with the recurrence of natural and buman disasters, and their effects on the populations' vulnerability, the Cameroonian government should implement long-lasting measures to support affected populations, rather than act sporadically, as is the case presently. Because while crises pass, their effects persist.
\end{abstract}

\section{Introduction}

Depuis des décennies, plusieurs pays africains vivent des situations de crise à répétition. Des situations dans lesquelles la vie d'un grand nombre de personnes est menacée, qu'il s'agisse de catastrophes naturelles (séismes, éruptions volcaniques, sécheresses, inondations, épidémies, etc.) ou de catastrophes humaines (conflits armés, incendies, accidents d'avion, etc.). Le Cameroun ne fait pas exception à cette nouvelle donne.

En effet, au cours des 40 dernières années, ce pays a fait face à des crises majeures, dont les catastrophes du lac Monoun (1984) et du lac Nyos (1986), l'incendie de Nsam conséquent à un accident ferroviaire (1998), l'insurrection de Boko Haram (depuis 2014), le déraillement du train de Camrail à Éséka (2016), les revendications armées dans les régions du NordOuest et du Sud-Ouest (depuis 2016), les inondations dans la région de l'Extrême-Nord (chaque année), l'éboulement à Gouache (2019) et la pandémie de COVID-19 (depuis mars 2020).

Au vu de leurs effets et de leur récurrence, ces crises ne peuvent plus être considérées comme des phénomènes épisodiques, comme c'est souvent le cas. Nous pensons qu'il faut désormais intégrer leur gestion en tant qu'activité courante de gouvernance nationale.

\section{Revue de littérature}

Les catastrophes ont fait l'objet de nombreuses recherches en sciences sociales (Fritz, 1961; Nudell et Antokol, 1988; Quarantelli, 1998; Alexander, 2000; OECD, 2003; Rodriguez et collab., 2007; Harissi et Tourne, 2020). Pour Quenet (2000), une catastrophe fait référence à un "événement qui échappe à la domination d'un groupe humain, dépassant sa capacité d'absorption - capacité technique, économique, culturelle ou mentale» (p. 17). Dans sa terminologie,

${ }^{a}$ Professeur titulaire, École d'administration des affaires, Université de Saint-Boniface, Winnipeg 
l'UNISDR (2009) appréhende la catastrophe comme «une rupture grave du fonctionnement d'une communauté ou d'une société impliquant d'importants impacts et pertes humaines, matérielles, économiques ou environnementales, que la communauté ou la société affectée ne peut surmonter avec ses seules ressources» (p. 11).

Les catastrophes peuvent être classées en deux grandes catégories : les catastrophes naturelles et les catastrophes technologiques. Les catastrophes naturelles comprennent les catastrophes hydrométéorologiques (inondations, ouragans, sécheresses et avalanches), les désastres géophysiques (tremblements de terre, éruptions volcaniques et tsunamis) et les catastrophes biologiques (épidémies et invasions d'insectes).

Quant aux catastrophes technologiques, elles sont associées aux accidents industriels (déversements de produits chimiques, explosions industrielles et radiations radioactives), aux accidents de transport (par voies aérienne, ferroviaire, maritime ou fluviale) et à divers autres accidents provoqués par l'humain (incendies, effondrements de structures, crimes ou guerres impliquant des populations civiles) (Nudell et Antokol, 1988; Moe et Pathranarakul, 2006).

Toutefois, cette typologie ne rend pas compte de l'imbrication entre les deux catégories. En fait, les catastrophes dites «naturelles» sont le plus souvent la conséquence d'actes d'omission et de commission humains, plutôt que de simples caprices de la nature. Bien plus, un désastre résulte de la coprésence de facteurs humains et naturels (Chmutina et Von Meding, 2019). Dans tous les cas, les catastrophes sont des phénomènes de grande amplitude dont les effets causent des dommages importants et laissent des séquelles à une communauté humaine. En ce sens, elles provoquent une rupture et mettent en évidence une série d'oppositions entre l'avant et l'après (Quenet, 2000).

Compte tenu de leurs impacts dans une communauté, tout un corpus de connaissances s'est développé autour de la gestion des catastrophes, des désastres ou des crises; trois termes que nous allons utiliser indifféremment dans cet article.

La gestion des catastrophes est un processus qui inclut l'ensemble des activités menées avant, pendant et après un désastre, et qui vise, d'une part, à éviter les pertes en vies humaines et les impacts économiques et, d'autre part, à assurer le retour à une vie normale (Maher, 2006). La gestion des catastrophes fournit des outils pour se préparer aux catastrophes, pour y répondre, pour apporter du soutien et pour reconstruire la société (Altay et Green, 2006). Elle comporte donc trois phases : la précrise, la phase d'urgence et la post-crise ou phase de reconstruction (Burnett, 1988; Kreps, 1991; Wybo, 2010).

\subsection{La précrise}

La précrise inclut toutes les actions à entreprendre en amont pour limiter les effets de la catastrophe lors de sa survenance. Il s'agit des actions de planification et de prévention engagées pour éviter et réduire les dommages éventuels causés par la survenance d'un désastre (Pielke et Pielke, 1997; Covington et Simpson, 2006). Dans la littérature, cette phase est surtout marquée par une recension des meilleures pratiques en matière de planification des actions à entreprendre au moment de la survenance d'une catastrophe (Dynes, 1994; McEntire, 2003; Lindell et Perry, 2003). En effet, il apparait que les effets d'une crise qui survient dans une communauté qui y est préparée sont généralement moindres que ceux d'une crise qui arrive par surprise (McEntire, 2003).

C'est pourquoi cette phase a fait l'objet d'une abondante publication, non seulement de la part des agences gouvernementales nationales telles que le National Research Council, la National Emergency Management Association et la Federal Emergency Management Agency aux États-Unis, mais aussi de la part des agences des Nations unies, dont le Cadre d'action de Hyogo pour (2005-2015) : pour des nations et des collectivités résilientes face aux catastrophes (UNISDR, 2005) et le Cadre d'action de Sendai pour la réduction des risques de catastrophe 2015-2030 (UNISDR, 2015). Ces deux cadres sont issus respectivement de la Conférence mondiale sur la prévention des catastrophes, qui s'est tenue du 18 au 22 janvier 2005 à Kobe (préfecture de Hyogo, au Japon) et de la Conférence mondiale de l'ONU tenue à Sendai au Japon le 18 mars 2015 sur le même sujet. Ce dernier entend «mettre un accent beaucoup plus soutenu sur la prévention, la gestion et la réduction des risques de catastrophe plutôt que sur la gestion des catastrophes une fois celles-ci survenues» (UNISDR, 2015, p. 5). Il consacre le rôle central que les gouvernements ont à jouer aux paliers national, régional et mondial, rôle qui tourne autour de quatre priorités : 1) comprendre les risques de catastrophe; 2) renforcer la gouvernance des risques 
de catastrophe pour mieux les gérer; 3) investir dans la réduction des risques de catastrophe aux fins de la résilience; et 4) renforcer l'état de préparation aux catastrophes pour intervenir de manière efficace et pour « mieux reconstruire » durant la phase de relèvement, de remise en état et de reconstruction.

De ce qui précède, nous pouvons émettre la proposition suivante:

Proposition 1: La gestion de l'étape de précrise est marquée, à l'échelle des États, par l'adoption d'instruments nationaux de prévention des catastrophes inspirés par les conventions internationales.

Si la responsabilité générale de réduire les risques de catastrophe incombe aux États, elle n'en est pas moins partagée entre les gouvernements et les parties prenantes de la société civile, à savoir les bénévoles, les organisations humanitaires et les associations communautaires (Sutton et Tierney, 2006). La collaboration entre l'État et ces parties prenantes s'étend aux phases subséquentes, à savoir la phase d'urgence (gestion de la crise) et la phase post-crise.

\subsection{La phase d'urgence}

La phase d'urgence est celle au cours de laquelle la réponse à l'urgence est mise en œuvre. L'objectif est de fournir une assistance prompte pour préserver des vies, pour améliorer la santé et pour apporter du soutien moral aux populations en détresse. Il est question de limiter les dommages causés par la catastrophe en attendant que des solutions plus pérennes soient implantées.

D'après Ben Massou (2011), cette phase nécessite le déploiement de trois types de moyens : les moyens techniques, les capacités organisationnelles et les aptitudes humaines. Au-delà de ces moyens, Linnell (2013) insiste sur l'importance de la communication et de la collaboration entre les acteurs.

Proposition 2: Dans la phase de crise, la communication est indispensable pour fédérer les énergies des acteurs et pour mieux faire face à la catastrophe.

La communication est indispensable avant, pendant et après la catastrophe (Linnell, 2013). Dans la phase de gestion de la crise proprement dite, elle est critique, car elle doit permettre de mettre au même diapason tous les acteurs impliqués dans une catastrophe. La communication doit être diversifiée aussi bien dans les médias utilisés que dans les audiences visées (Turner et Muller, 2004; Sellnow et Sellnow, 2010). C'est ainsi qu'elle accroit la participation et la collaboration des parties prenantes (Newport et Jawahar, 2003; NAS, 2011). Cette collaboration est essentielle entre ceux qui sont chargés de la gestion de la crise, la population en général et les organisations communautaires. En effet, d'après la Federal Emergency Management Agency (FEMA, 2011) aux États-Unis, la mobilisation des individus et des groupes réussit mieux lorsqu'elle s'appuie sur des réseaux établis.

\subsection{La post-crise ou phase de reconstruction}

La post-crise est une phase de reconstruction et de gestion des séquelles de la catastrophe. C'est la phase de réadaptation aux effets de la crise, tant sur les plans de la construction et de la réhabilitation des infrastructures que de la mise en place de mesures d'atténuation des effets socioéconomiques de la catastrophe sur les populations.

La post-crise est présentée comme une étape très complexe qui s'étend généralement sur plusieurs années (Labadie, 2008). Malheureusement, malgré la multiplicité des expériences, cette phase reste insuffisamment documentée (Halvorson et Hamilton, 2010).

Une question se pose ici : la reconstruction doit-elle remettre en état les choses telles qu'elles étaient avant la catastrophe ou faut-il plutôt changer en mieux (build back better)? Pour Lyons (2009), la première option permet de préserver le patrimoine, mais elle a le désavantage de reproduire les mêmes conditions de vulnérabilité auxquelles la population était exposée avant la catastrophe. Dans la seconde option, la postcrise donne l'occasion de corriger les facteurs de vulnérabilité propices à la survenance des catastrophes semblables dans le futur et d'y remédier (Lewis, 2003; Kijewski-Correa et Taflanidis, 2012). Mieux: elle donne la possibilité d'utiliser le processus de reconstruction pour améliorer les conditions physiques, sociales, environnementales et économiques d'une communauté et pour la rendre plus résiliente (Clinton, 2006). Cependant, toute phase de reconstruction exige de disposer de ressources (Xie, 1994; Ye et Okada, 2002).

Pour Chang et ses collègues (2011), la disponibilité des ressources (financières, humaines, managériales et matérielles) est indispensable à la réussite de tout 
projet de reconstruction, comme le sont tout autant les facteurs administratifs, économiques, politiques, sociaux et culturels. La non-prise en considération de ces éléments affecte directement la vitesse et les résultats du processus de reconstruction (Shaw, 2006). Ce dernier se présente comme un projet collectif qui doit associer les différentes parties prenantes. Pour Shafique et Warren (2016), la première étape dans la phase de reconstruction est donc d'identifier les acteurs qui comptent. Ceux-ci se recrutent parmi les agences gouvernementales, les organisations non gouvernementales (ONG; nationales, régionales ou internationales), les médias, le secteur privé, la communauté prise dans son sens large, etc. (Chandrasekhar, 2012).

Proposition 3 : Compte tenu de sa complexité et de sa durée, la phase de reconstruction exige une mobilisation plus importante de ressources et une implication plus grande de la communauté et des organisations de première ligne.

L'implication de la communauté et des ONG est une condition de succès de la phase de reconstruction (Shaban, 2001; Linnell, 2013; Miller et Douglass, 2016). Mieux: l'action coordonnée des différents intervenants accroitt l'efficacité de l'action de reconstruction sur le terrain (Meduri, 2016). Peut-on en dire autant du Cameroun?

\section{Gestion de la pandémie au Cameroun : état des lieux}

L'épidémie de la COVID-19 est apparue pour la première fois dans la ville de Wuhan en Chine en décembre 2019. Elle a été déclarée comme pandémie le 11 mars 2020 par l'Organisation mondiale de la santé (OMS). En date du 30 mars 2020, elle avait déjà fait 33106 décès dans 202 pays et territoires.

Le premier cas en Afrique subsaharienne est apparu le 27 février 2020 au Nigéria. Depuis lors, les chiffres se sont emballés. Au 25 août 2020, on comptait à travers le monde plus de 23689860 cas d'infection officiellement diagnostiqués depuis le début de la pandémie, dont 813733 morts. Les États-Unis étaient alors considérés comme le pays le plus touché, avec 177284 décès et plus de 5741088 cas recensés (Agence France-Presse, 2020a).
Au Cameroun, le premier cas a été déclaré le 6 mars 2020. Depuis lors, la pandémie a connu une progression fulgurante : elle est passée de 142 cas testés positifs et 6 morts le 30 mars 2020 à 7860 cas positifs et 215 morts le 7 juin 2020, puis à 12592 personnes testées positives, 10100 personnes déclarées guéries et 313 décès à la fin du mois de juin 20201. Cette progression faisait de ce pays l'un des plus atteints par la pandémie en Afrique.

Pour faire face à la pandémie, le gouvernement a pris une série de mesures qui peuvent être classées en trois catégories : les mesures stratégiques, les mesures opérationnelles et les mesures à l'endroit de la population en général.

\subsection{Les mesures stratégiques}

Près de trois mois après l'apparition du coronavirus en Chine et au vu de sa propagation dans les autres pays, notamment en Europe, le gouvernement camerounais a mis en place, à partir de février 2020, le Plan de préparation et de réponse au COVID-19 d'un montant de 6,5 milliards FCFA (soit 11,4 M\$ US). D’après le ministre camerounais de la Santé, le plan en question « est un document-cadre qui déroule les composantes essentielles, selon l'OMS, dont les actions prioritaires sont à mener dans les 30, 60 et 90 prochains jours » (Ministère de la Santé publique, 2020, p. 3). L'objectif général de ce plan vise à détecter précocement les éventuels cas importés de COVID-19 au Cameroun et à y apporter une riposte efficace, le cas échéant.

Pour son élaboration, le Plan de préparation et de réponse an COV ID-19 est le fruit de la collaboration entre les experts du ministère de la Santé publique, ceux des autres ministères les plus impliqués dans la gestion de la COVID-19, ceux de l'OMS et des autres partenaires multilatéraux.

En matière de contenu, le plan se décline en huit axes : 1) la coordination multisectorielle et internationale; 2) la surveillance pour la détection précoce des cas; 3) la mise sur pied des équipes d'enquête et d'intervention rapide; 4) le renforcement des capacités des laboratoires; 5) la mise en place des mesures de prévention et de contrôle des infections dans les hôpitaux et les communautés; 6) la gestion des cas; 7) la communication des risques et l'engagement communautaire; et 8) le soutien logistique. 
Le plan en question est par ailleurs mis en œuvre à travers un plan national opérationnel de préparation et de réponse au COVID-19 (Ministère de la Santé publique, 2020, p. 14).

\subsection{Les mesures opérationnelles}

Les mesures opérationnelles se déploient à travers le Système de gestion de l'incident (SGI), dont la coordination centrale se fait au sein du ministère de la Santé publique et dont les démembrements territoriaux suivent l'organisation administrative du pays (régions, départements, etc.). D’après la docteure Djamilatou Leila, déléguée régionale de la santé publique de la Région du Nord:

L'objectif de l'activation du SGI vise : la détection précoce de tous cas suspects; d'assurer le diagnostic dans de brefs délais; assurer la prise en charge immédiate d'éventuels cas; assurer la mise en place des mesures de prévention et contrôle de l'infection; assurer une bonne coordination multisectorielle des activités de préparation et de réponse et une bonne information de la population et des différents détenteurs d'enjeux nationaux et internationaux. (Mbeng Boum, 2020, p. 8)

Par ailleurs, pour faire face à une saturation éventuelle des hôpitaux dédiés à la lutte contre la COVID-19, des centres spécialisés ont été mis en place pour la prise en charge des victimes de la pandémie dans les grandes villes, notamment Yaoundé, Douala et Bafoussam.

Pour financer ces diverses opérations, le gouvernement a annoncé, le 30 mars 2020, la création par le président de la République d'un fonds de solidarité avec une dotation initiale de 1 milliard FCFA (soit $1,7 \mathrm{M} \$$ US). Ce fonds est alimenté par des contributions de toutes natures provenant des entreprises, des organisations et des personnes de bonne volonté. Cette mesure est étendue par le président de la République à travers l'Ordonnance no 2020/001 du 03 juin 2020 modifiant et complétant certaines dispositions de la loi n 2019/023 du 24 décembre 2019 portant loi de finances de la République du Cameroun pour l'exercice 2020, laquelle :

- Accorde une exonération totale des droits et taxes de douane à l'importation de produits et matériels de prévention et de lutte contre la COVID-19 (art. 13 bis);
- Pour soutenir l'activité économique, suspend pour une période de 6 mois la perception des intérêts de retard au paiement des droits et taxes de douane (art. 14 bis);

- Rend totalement déductibles, pour le calcul de l'impôt sur les sociétés, les dons et libéralités consentis à l'État ou ses démembrements, dans le cadre de la lutte contre la pandémie de la COVID-19 (art. 15 bis);

- Institue l'ouverture d'un compte d'affectation spécial intitulé «Fonds spécial de solidarité nationale pour la lutte contre le coronavirus et ses répercussions économiques et sociales» (art. 26 bis). (Ministère des Finances, 2020 b, p. 2)

Le fonds ci-dessus a pour objet la prise en charge des besoins liés à la lutte contre la pandémie du coronavirus au Cameroun, ainsi que ceux relatifs à l'intervention de l'État dans le cadre de l'atténuation des conséquences économiques, financières et sociales de cette pandémie, et des mesures restrictives prises par le gouvernement. Au second semestre de 2020, le Fonds a reçu une dotation de 180 milliards FCFA (soit environ 317 M\$ US) répartis entre les différentes structures gouvernementales impliquées dans la lutte et la prévention de la COVID-19 (autant dire presque tous les ministères).

D’après le Décret n ${ }^{\circ}$ 2020/3221 du 22 juillet 2020 fixant la répartition de la dotation du Fonds spécial de solidarité nationale pour la lutte contre le coronavirus et ses répercussions économiques et sociales (Ministère des Finances, 2020a), les dépenses supportées par ledit fonds concernent la recherche active des cas et la prise en charge des cas confirmés; la couverture des dépenses liées aux mesures administratives et de régulation sociale; l'acquisition des intrants; l'encadrement des producteurs; et la mise en place du dispositif d'incitations pour une meilleure production agricole, vivrière et pastorale (notamment les produits de grande consommation et de substitution susceptibles de réduire la dépendance alimentaire). Le fonds devrait également servir au développement de la recherche et de la production locale des produits pharmaceutiques de première nécessité; au développement des enseignements à distance et à la distribution d'outils et supports pédagogiques en milieu rural; et à l'octroi des appuis de soutien aux personnes socialement vulnérables et aux enfants de la rue affectés par la COVID-19. 
Outre le fonds, le gouvernement camerounais reçoit des dons en nature de toutes sortes (matériel médical, masques, produits d'hygiène et désinfectants, denrées alimentaires, etc.) destinés à la population en général.

\subsection{Les mesures à l'endroit de la population en général}

Les mesures vis-à-vis des populations ont pour la plupart été édictées par l'Organisation mondiale de la santé. Au Cameroun, elles ont été mises en place à partir du 18 mars 2020. Elles allaient de la fermeture des frontières terrestres, aériennes et maritimes, des débits de boissons et autres lieux de loisirs à partir de 18 h, à l'arrêt des cours dans les établissements scolaires relevant des trois ordres d'enseignement (primaire, secondaire et supérieur), en passant par l'annulation de toutes les compétitions sportives, l'interdiction des rassemblements publics de plus de 50 personnes, la limitation des déplacements urbains et interurbains, etc. Un numéro de téléphone pour mobiliser les équipes de secours (le 1510) était également à la disposition de la population.

Le gouvernement camerounais a aussi encouragé le recours à la médecine traditionnelle à travers un train d'annonces, mais surtout en laissant la population «se débrouiller». Le ton des annonces a été donné par le président de la République Paul Biya qui, dans son discours du 19 mai 2020, a affirmé qu’il « encourage également tous les efforts visant à mettre au point un traitement endogène du COVID-19» (CRTV, 2020).

Pour matérialiser cette invitation, le premier ministre Joseph Dion Ngute a rendu publiques un ensemble de propositions, dont l'encadrement des initiatives développées à partir de la pharmacopée traditionnelle; l'examen minutieux des solutions thérapeutiques proposées par les tradipraticiens; le renforcement de la collaboration entre le ministère de la Santé publique, le ministère de la Recherche scientifique et de l'Innovation, le ministère de l'Enseignement supérieur et les tradipraticiens. Dans la même veine, le ministère de la Santé publique s'est engagé à accélérer le processus de finalisation d'un projet de loi portant organisation et fonctionnement de la médecine traditionnelle au Cameroun, de même que la mise en place d'un répertoire national des tradithérapeutes de santé (Manaouda, 2020; Mbwe Mpoh et Briand K., 2000). Le tableau ci-dessous présente quelques-uns de ces tradithérapeutes de la COVID-19 et leurs inventions.

\begin{tabular}{|l|l|l|}
\hline Nom du produit & \multicolumn{1}{|c|}{ Inventeur } & \multicolumn{1}{c|}{ Fonction } \\
\hline Soudicov plus & Cheick Modibbo Halidou Ibrahim & Tradipraticien \\
\hline Efu_eyoh_koh & Acha Vincent Njeck & Tradipraticien \\
\hline Ngul be tara & Dr Peyou Ndi Marlyse & Enseignant-chercheur \\
\hline Viro-green F.4 & Dre Joséphine Briand K & Naturopathe \\
\hline $\begin{array}{l}\text { Elixir-COVID } \\
\text { Adsak-COVID }\end{array}$ & Mgr Samuel Kleda & Archevêque \\
\hline Tucloxine & Dharous Salam & Tradipraticien \\
\hline Bio covax & Mme Njikam, la reine des abeilles & Tradipraticienne \\
\hline Jatrophicine & Dr Temfack Vope & Enseignant-chercheur \\
\hline
\end{tabular}

Tableau 1 - Quelques pistes de solution endogènes proposées par des praticiens et chercheurs de la médecine traditionnelle du Cameroun Source : Mbwe Mpoh, 2020, p. 28

En bonne place dans ce répertoire des tradithérapeutes figure Mgr Samuel Kleda, l'archevêque métropolitain de Douala, dont l'initiative de la mise au point d'une potion censée guérir la COVID-19 illustre l'option du gouvernement à laisser la population se «débrouiller». D'après l'hebdomadaire Jeune Afrique (Foute, 2020a), Mgr Kleda s'intéresse à la phytothérapie depuis bientôt 30 ans. À Douala, il a ouvert une herboristerie où se font soigner plusieurs patients. Mgr Kleda affirme avoir mis au point une thérapie à base de plantes capable de soigner la COVID-19.
Pour encadrer son initiative, le ministère de la Santé publique a mis sur pied une plateforme technique. De même, plusieurs personnalités lui ont offert des fonds pour produire et distribuer à grande échelle son élixir.

Cependant, certains professionnels de la santé reprochent au ministère de la Santé publique d'avoir laissé faire l'archevêque de Douala sans attendre les conclusions de la plateforme technique. Environ quatre mois après avoir largement distribué son traitement à des milliers de personnes, Mgr Kleda annonçait à ses 
fidèles, le $1^{\text {er }}$ août 2020, la fin de la guerre qu'il mène contre la COVID-19 :

Nous avons vaincu le coronavirus. Le stock des remèdes contre cette pandémie chôme depuis quelques jours dans nos hôpitaux catholiques, car il n'y a presque plus de malades. Nous rendons grâce à Dieu et à la très Sainte Vierge Marie pour cette merveille. [...] Le coronavirus n'est plus une menace au Cameroun. (Ntchapda, 2020, s. p.)

C'est sur cette déclaration de foi, qui n'a fait l'objet d'aucune réaction de la part du ministère de la Santé publique, qu'il convient de s'arrêter pour tirer les premières leçons de la gestion de la COVID-19 au Cameroun.

\section{Premières leçons à tirer de la gestion de la pandémie}

Dans cette partie, nous nous intéressons à la phase de précrise de pandémie de COVID-19 et à la phase de gestion de l'urgence, qui est actuellement mise en œuvre au moment d'écrire ces lignes, avant de nous interroger sur ce qu'il en sera de la phase de post-crise.

\subsection{La gestion de la précrise : le déficit de mise en cohérence de l'armature institutionnelle}

La phase de précrise est marquée, de façon générale, par l'adoption d'instruments nationaux de prévention des catastrophes inspirés des conventions internationales. Au Cameroun, le ministère de l'Administration territoriale en est le principal acteur. D’après Tchingankong Yanou (2014), ce choix date de 1961 avec la création, au sein de la Direction des affaires politiques de ce ministère, du Service de la protection civile, chargé de la gestion des sinistres. Ce service sera érigé plus tard, en 1995, en Direction de la protection civile (DPC). La DPC fonctionne en synergie avec d'autres organisations telles que la Croix-Rouge camerounaise, le ministère de la Santé publique, le Corps national des sapeurspompiers, le Service d'assistance médicale d'urgence, l'Institut des recherches géologiques et minières, etc.

Au fil des catastrophes, l'armature institutionnelle au Cameroun s'est étoffée, généralement dans le but de rendre plus efficaces les interventions gouvernemen- tales. Dans cette optique, l'État a créé d'autres structures, dont le Conseil national de la protection civile (CNPC) en 1996, l'Organisation des plans d'urgence et des secours en cas de catastrophe ou de risque majeur (ORSEC) en 1998, le Programme national de prévention et de gestion des catastrophes (PNPGC) en 1998 et l'Observatoire national des risques (ONR) en 2003 .

\section{Le Conseil national de la protection civile (CNPC)}

D’après le Décret no 96/054 du 12 mars 1996 fixant la composition et les attributions du Conseil national de la protection civile (MINATD, 1996), le CNPC est un organisme consultatif qui assiste le président de la République en matière de protection civile. Il doit veiller entre autres à :

- une évaluation nationale détaillée des risques de catastrophes naturelles et technologiques, d'accidents graves et de calamités;

- à la mise à jour permanente d'un inventaire de fournitures, de matériels, de moyens et de personnels pouvant être mobilisés en cas de situation d'urgence;

- aux études générales sur les mesures de protection civile en temps de paix comme en temps de guerre» (paragr. 5[1]).

Par ailleurs, le Conseil est chargé de l'élaboration d'un plan national d'intervention et d'organisation (art. 6).

L'Organisation des plans d'urgence et des secours en cas de catastrophe on de risque majeur (ORSEC)

À la suite de l'incendie de Nsam conséquent à un accident ferroviaire, un plan d'organisation des secours par l'ORSEC a vu le jour par le Décret $n^{\circ}$ 98/031 du 09 mars 1998 portant organisation des plans d'urgence et des secours en cas de catastrophe ou de risque majeur (MINATD, 1998). Il s'agit d'un ensemble des mesures d'intervention rapide qui doivent être prises pour faire face à des catastrophes ou à des risques majeurs survenant sur l'étendue du territoire camerounais. Les plans de l'ORSEC sont un «dispositif d'organisation territorialisée de la gestion des sinistres. Ils placent les autorités administratives en situation de mobilisation de l'ensemble des ressources nécessaires pour la maitrise des phénomènes inopinés » (Tchingankong Yanou, 2014, p. 110). Ainsi, le déclenchement des plans d'urgence et des secours en cas de catastrophe ou de risque majeur se fait par le préfet à l'échelle du département, par le gouverneur à l'échelle 
de la région et par le secrétaire général de la présidence de la République au palier national.

\section{Le Programme national de prévention et de gestion des catastrophes (PNPGC)}

Pour ce qui est du PNPGC, il a été mis en place au Cameroun en 1998 avec la collaboration du Programme des Nations unies pour le développement (PNUD) à la suite de la Conférence mondiale sur la prévention et la gestion des catastrophes tenue à Yokohama, au Japon, en mai 1994. Le PNPGC a pour objectif le renforcement des capacités managériales, matérielles et logistiques du gouvernement en matière de planification, de prévention et de gestion des catastrophes (DPC, 2003).

\section{L'Observatoire national des risques (ONR)}

Quant à l'Observatoire national des risques (ONR), il est régi par l'Arrêté n' 037/PM du 19 mars 2003 portant création, organisation et fonctionnement d'un observatoire national des risques (Premier ministre du Cameroun, 2003). Il a pour mission la collecte, la gestion et la diffusion des informations sur les risques naturels et technologiques. C'est un «cadre de concertation et de collaboration entre les différentes administrations concernées, les organismes publics ou privés, nationaux et internationaux impliqués dans la gestion préventive des risques» (paragr. 2[2]).

L'armature institutionnelle en phase de précrise est donc bel et bien en place au Cameroun. Cependant, elle brille par son instabilité du fait qu'elle change au gré de la survenance des catastrophes.

Par ailleurs, elle comporte un certain nombre d'obstacles qui sont susceptibles de limiter l'action du gouvernement en matière de gestion des catastrophes. Au premier rang de ces limites, il y a la multiplicité des textes et des acteurs. Puisque les catastrophes sont des problèmes transversaux, cette multiplicité augmente les coûts d'intervention et empêche la concentration de l'expertise au même endroit.

La gestion des catastrophes au Cameroun fait par ailleurs la part belle au ministère de l'Administration territoriale et de la Décentralisation (MINATD) et à sa Direction de la protection civile (DPC). Certes, cette dévolution est susceptible d'induire une cohérence dans l'action, mais il reste que ce ministère est politiquement marqué (par le parti au pouvoir), ce qui entraîne une méfiance d'une partie de la population quant à l'adhésion de ses décisions ${ }^{2}$.

Plus généralement, hormis la Croix-Rouge, la gestion des crises au Cameroun est caractérisée par un quasimonopole des fonctionnaires. Les textes régissant ce domaine font très peu de place aux représentants et aux organismes de la société civile. Or, dans le contexte d'un pays où les fonctionnaires changent constamment de postes et de ministères, il est difficile de développer un apprentissage organisationnel en matière de gestion des situations d'urgence.

\subsection{La gestion de la phase d'urgence}

Cette phase est marquée par un certain nombre de dysfonctionnements propres à la gestion publique au Cameroun, à savoir le règne du gradualisme disjoint dans la prise de décision, la prépondérance d'une approche bureaucratique, les conflits de compétences et un déficit de communication.

\subsubsection{Le règne du gradualisme disjoint}

Le gradualisme disjoint (ou la politique des petits pas) est un mode de prise de décision mis de l'avant par Lindblom (1959). Il consiste en une suite d'approximations visant à résoudre un problème. Ainsi, on avance à petits pas en apprenant dans le cours de l'action. La gestion de la pandémie de COVID-19 au Cameroun illustre à merveille cette théorie.

En effet, même s'il est encore très tôt pour faire le bilan de la réponse du gouvernement camerounais face à la pandémie de COVID-19, on peut affirmer que la gestion de cette pandémie a connu, dans ses débuts, beaucoup de tâtonnements, la démarche du gouvernement s'apparentant à une sorte de « bricolage permanent » (Tchingankong Yanou, 2014). Par la suite, le gouvernement s'est ressaisi en appliquant autant que possible les mesures préconisées par l'OMS en pareille circonstance. En effet, l'OMS a publié un document qui fournit aux États membres les orientations provisoires pour la mise en œuvre de la surveillance mondiale du nouveau coronavirus COVID-19 (OMS, 2020). Malgré ces points à mettre à l'actif du gouvernement camerounais, certaines lacunes peuvent être évoquées dans sa gestion de cette pandémie. 


\section{L'incapacité du gowvernement à empêcher la propagation de la maladie en début de pandémie}

La première lacune est relative à l'incapacité du gouvernement à empêcher la propagation de la maladie au tout début de la pandémie. En effet, le début de la propagation du coronavirus au Cameroun est présenté par bien des observateurs comme étant le résultat du laxisme qui a régné dans l'application des contrôles aux aéroports. Ce laxisme est déploré dans cet extrait du journal Le Monde Afrique du 16 mars 2020 :

De nombreuses voix s'élèvent pour réclamer l'arrêt des liaisons aériennes et maritimes avec les pays qui dénombrent le plus grand nombre de malades comme la France et l'Italie, mais le gouvernement n'en est pas encore là. Dans un communiqué paru dimanche 15 mars, le ministre de la Santé publique, le docteur Manaouda Malachie, invite plutôt les passagers des vols Air France n ${ }^{\circ}$ AF 900 et SN Brussels $n^{\circ} 371$ ayant transporté deux des cinq patients «à bien vouloir se signaler de toute urgence». Et dans un autre communiqué paru ce 16 mars, M. Malachie invite tout voyageur en provenance des pays à risque à « observer une quarantaine de quatorze jours avant toute activité et [tout] contact». (Le Monde Afrique, 2020, s. p.)

\section{L'impuissance du gouvernement à faire respecter les mesures édictées}

La deuxième lacune a trait à l'impuissance du gouvernement à faire respecter par la population les mesures barrières qui ont été édictées. En effet, le 30 avril 2020, le gouvernement camerounais a décidé de lever certaines mesures de distanciation sociale dans tout le pays afin de soulager les secteurs fortement affectés par la crise sanitaire. Il s'agissait de l'ouverture au-delà de $18 \mathrm{~h}$ des débits de boissons, des restaurants et des lieux de loisirs ainsi que de la levée de la mesure réduisant le nombre de passagers dans tous les transports en commun. Cette annonce du gouvernement a été considérée par la population comme signifiant la fin de la pandémie. On a alors observé une multiplication des comportements à risque :

«C'est terrible, regardez, regardez, c'est le désordre tous azimuts et les gens se sont accoutumés à ça, ils se sont habitués à ça et ça ne leur dit rien. C'est leur train-train quotidien. Regardez dans les bars, les gens s'embrassent, se parlent bouche à bouche avec des postillons qui sortent d'une bouche à l'autre, ça ne dit rien à personne», témoigne Claude Epoh, ingénieur en construction. (Lyonnel, 2020, s. p.)

Dans un pays où la majorité de la population vit dans la promiscuité et au jour le jour, de tels comportements ne surprennent pas.

Le manque de moyens du gouvernement et des populations en général

La troisième lacune est inhérente à un manque de moyens à la fois du gouvernement et des populations en général. La pandémie de COVID-19 est arrivée au moment où le pays faisait face à une situation économique difficile marquée par une baisse des ressources de l'État. D'après Savina Ammassari, directrice pays de l'ONUSIDA pour le Cameroun :

La riposte du Cameroun est confrontée à des contraintes considérables, en particulier pour ce qui est d'élargir le dépistage et le traçage des contacts, de fournir des unités de soins intensifs/des respirateurs artificiels pour les personnes gravement atteintes, ainsi que des équipements de protection individuelle adaptés destinés au personnel médical. La mise en place rapide de programmes d'éducation des communautés mettant l'accent sur l'hygiène des mains et l'éloignement physique est une problématique actuelle, en particulier dans les zones urbaines pauvres et souvent surpeuplées (ONUSIDA, 2020, s. p.).

Au-delà de ces lacunes, qui peuvent être classées comme conjoncturelles, d'autres limites marquent la gestion de la pandémie de COVID-19 au Cameroun. Ces limites peuvent être qualifiées de structurelles, dans la mesure où elles sont récurrentes chaque fois que le pays fait face à une catastrophe. Elles ont trait à la nature de l'intervention gouvernementale, aux conflits de compétences entre les organes étatiques, au déficit de transparence dans la gestion des dons et à l'absence d'anticipation du gouvernement camerounais. 


\subsubsection{Une gestion bureaucratique et exclusive de la pandémie par les organes étatiques}

La gestion de la pandémie au Cameroun a été marquée par une prépondérance des interventions gouvernementales. Cette gestion est ponctuée d'ordonnances, de décrets, d'arrêtés et d'une diversité d'instances de concertation gouvernementales.

La coordination générale de la réponse à la pandémie est assurée par le premier ministre. Compte tenu de l'ampleur de la menace, cette implication du gouvernement se comprend. Ce qui est à déplorer, c'est la centralisation de la prise de décision au palier ministériel et la quasi-exclusion des organes non étatiques de ce processus. Ainsi en est-il du don matériel d'une valeur de 2 milliards FCFA du président de la République, dont le cheminement est décrit par le MINATD ${ }^{3}$ en ces termes : «Le chef de l'État m'a instruit [le ministre] de venir lancer ce convoi. Les gouverneurs de régions vont prendre le relai, ainsi que les préfets, les sous-préfets et les maires » (Pahai et Toussaint, 2020, s. p.).

On sait que les premières heures d'une catastrophe sont critiques pour limiter l'amplification des effets. Dans le cas de la COVID-19, les errements du gouvernement camerounais au début de la pandémie ont amplifié sa propagation au sein de la population :

De fait, le Cameroun n'a fermé ses frontières terrestres, aériennes et maritimes que le 18 mars, douze jours après le premier cas «importé» et des semaines après nombre d'autres pays africains. [...] Il a fallu attendre mi-mars, aussi, pour que d'autres mesures de restriction soient prises, comme la distanciation dans les transports publics et la fermeture des bars et restaurants. (Agence France-Presse, 2020b, s. p.)

Nulle part dans la stratégie du gouvernement il n'est fait allusion à la participation des organisations non gouvernementales (ONG) locales, pourtant censées être spécialisées dans le soutien aux populations vulnérables. Seules les ONG internationales sont impliquées à travers du financement reçu de l'extérieur. Par exemple, l'ONG internationale Action contre la faim a reçu $11 \mathrm{M} €$ de l'Agence française de développement pour améliorer les conditions d'accueil et de prise en charge des personnes contaminées dans les hôpitaux de référence (Agence française de développement, 2020).

\subsubsection{Des conflits de compétences entre les organes étatiques}

La gestion de la pandémie a mis en branle un ensemble d'acteurs dont la délimitation des champs de compétences reste floue. Si les dévolutions du ministère de la Santé publique vont de soi dans la gestion de cette crise, la place prépondérante prise par le ministère de l'Administration territoriale s'explique moins, d'autant plus que le ministère des Affaires sociales et celui de la Décentralisation et du Développement local (chargés des collectivités locales) semblent mis de côté. Le ministre de l'Administration territoriale sillonne le pays d'est en ouest, du nord au sud pour la distribution des dons aux populations, et ces dons sont présentés comme étant ceux du président de la République.

On note les mêmes interférences en matière de communication. Entre le premier ministre, le ministre de la Santé publique et celui de la Communication, il est difficile de savoir qui dirige la communication gouvernementale en situation de crise. Très peu de médias privés ont été sollicités pour appuyer cette communication.

\subsubsection{Un déficit de communication et de tranparence dans la gestion des dons}

Bien des acteurs de la société civile se sont interrogés sur la transparence qui entoure l'utilisation des fonds destinés à la lutte contre la COVID-19. Ici encore transparaissent les lacunes du gouvernement en matière de communication. Le 12 juin 2020, l'ONG Human Right Watch (HRW), qui a pignon sur rue au Cameroun, a demandé qu'une enquête soit ouverte sur l'utilisation du Fonds de solidarité pour la santé (à ne pas confondre avec le Fonds de solidarité créé par le président de la République le 30 mars 2020). Ce fonds «a été mis en place pour résoudre les problèmes de santé urgents et garantir l'équité dans le système de santé; cependant, son utilisation n'est encore régie par aucune législation » (Banque mondiale, 2018, p. 131). Créé en 1993 par les pouvoirs publics, ce fonds est alimenté par une contribution de $10 \%$ des recettes mensuelles des établissements publics fournissant des soins médicaux primaires (HRW, 2020). 
Selon HRW et la Banque mondiale, il n'y a aucune traçabilité des fonds reçus ni des dépenses éventuellement engagées, ce qui laisse présumer une mauvaise utilisation des encaissements ou, pire, des détournements par corruption.

Les mêmes soupçons pèsent sur la gestion des dons reçus dans le cadre de la pandémie. D'après l'hebdomadaire Jeune Afrique, ces soupçons portent sur cinq enveloppes budgétaires :

Il s'agit principalement du fonds de solidarité créé par Paul Biya, initialement renfloué par ses soins à hauteur d'un milliard de francs CFA et dont le montant dépasserait déjà les 3 milliards de francs CFA; d'une facilité de 135,56 milliards de francs CFA, soit 226 millions de dollars, accordée par le FMI; des dons en nature d'entreprises et d'organisations diverses; du compte d'affectation budgétaire de 180 milliards de francs CFA adopté par l'Assemblée nationale; et enfin du Fonds de solidarité pour la santé, qui reçoit, depuis sa création en 1993, $10 \%$ des recettes mensuelles en provenance des établissements publics fournissant des soins médicaux primaires. Un total d'au moins 318,56 milliards de francs CFA auquel s'ajoutent les dons en nature. (Foute, 2020b, s. p.)

Face à cette situation, le premier ministre camerounais annonce l'ouverture d'une enquête sur l'utilisation des fonds alloués à la lutte contre la COVID-19, enquête qui sera menée par la Chambre des comptes.

\subsection{Quid de la phase de reconstruction?}

Cette phase n'est pas encore d'actualité, mais, au regard des expériences passées, il est facile d'imaginer qu'elle ne sera pas différente de la façon dont les autres catastrophes ont été gérées. En effet, d'après Ben Tchinda Ngoumela (2010), les membres de la protection civile ne sont visibles que lors de la survenance des catastrophes.
La phase d'urgence terminée, probablement du fait qu'elle est la plus médiatisée, les populations se retrouvent livrées à elles-mêmes. Ce quasi-abandon des populations a été observé lors des catastrophes précédentes. Or, avec la pandémie, le gouvernement camerounais ne peut se permettre une telle attitude. Du fait de son ampleur et de ses effets à long terme, la pandémie se situe dans un registre à part dans la chronique des catastrophes. Qui plus est, la future survenance d'autres pandémies de même nature et de même ampleur devient de plus en plus probable (Buissonnière, 2012; OMS, 2018).

Pour ce qui est de la pandémie de COVID-19, le Fonds monétaire international (2020) affirme: «Alors que le nombre de cas de COVID-19 continue d'augmenter, l'économie mondiale se prépare à un choc d'une gravité et d'une complexité sans précédent, qui devrait déclencher la pire récession depuis la Grande Dépression » (cité dans Valensisi, 2020, p. 1). Aussi, la Banque mondiale (2020) recommande-t-elle :

La première des priorités est de faire face à l'urgence sanitaire et économique mondiale. Mais, au-delà de ça, la communauté internationale doit s'unir pour trouver les solutions qui permettront de réinstaller une reprise aussi solide que possible et de lutter contre une aggravation de la pauvreté et du chômage. (s. p.)

Pour ce qui est du Cameroun, le Programme des Nations unies pour le développement (2020) estime que la pandémie de COVID-19 aura des conséquences importantes qui se traduiront par une baisse des recettes de l'État et par une augmentation de ses dépenses. Celles-ci engendreront à leur tour une baisse de production et du chiffre d'affaires des entreprises, ce qui aura des effets sur les ménages en matière de baisse des revenus, d'accroissement des dépenses de santé et d'augmentation du chômage. Ce système crée un cercle vicieux qui aggrave sans cesse ces effets néfastes, comme il apparait dans la figure 1 : 


\section{Ecconomie camerounaise : baisse de la croissance}

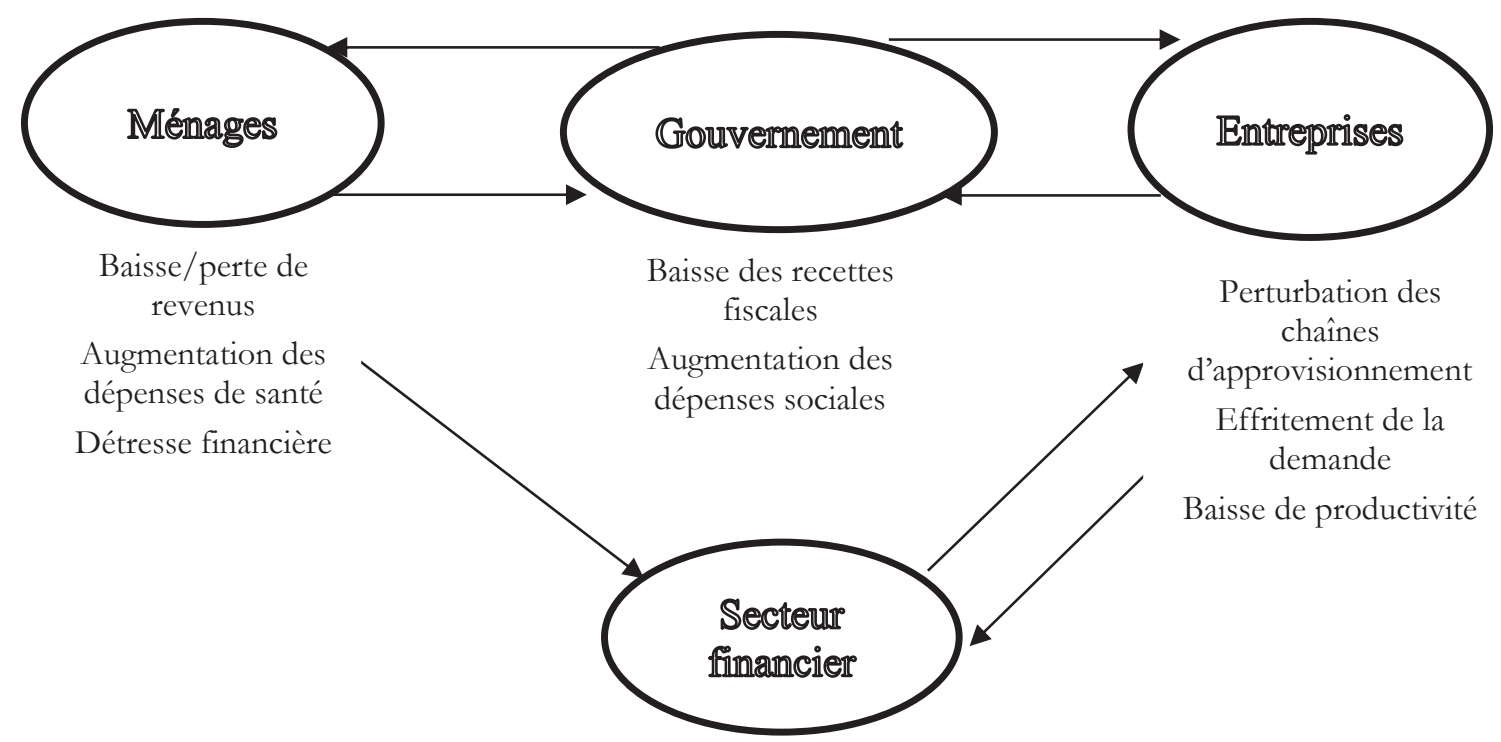

Risque de crise bancaire (retrait massif, non-paiement de crédits, réduction d'épargne interne, baisse des transferts internationaux, etc.)

Figure 1 - Effets socioéconomiques potentiels de la pandémie de COVID-19 au Cameroun Source : Adapté de PNUD, 2020, p. 11

Le schéma ci-dessus illustre l'ampleur des défis à venir une fois que la phase d'urgence de la pandémie aura été maitrisée. Dans ce cas précis, le gouvernement camerounais ne pourra pas baisser les bras comme il l'a souvent fait pour les autres catastrophes. En fait, à partir des lacunes relevées dans le système de gestion actuel de la pandémie de COVID-19, il faut repenser le processus de gestion des catastrophes au Cameroun en accroissant ses moyens et ses capacités d'intervention.

\section{Amélioration de la gestion des catastrophes}

Au vu des expériences passées et du cas de la pandémie de COVID-19, nous proposons que soit revue la façon d'aborder la gestion des catastrophes au Cameroun. Cela implique d'intégrer la gestion des catastrophes comme relevant des activités courantes de gouvernance du pays ainsi que de développer les capacités et les moyens d'intervention du système.

\subsection{Améliorer la gouvernance en matière de gestion des catastrophes}

Dans ce contexte, améliorer la gouvernance signifie de mettre en cohérence l'armature institutionnelle de gestion des crises. Cela suppose de faire une relecture et une mise à jour des textes organisant la gestion des catastrophes au Cameroun afin de mieux répartir les rôles, d'une part, entre les organes étatiques et, d'autre part, les acteurs privés et la société civile. Cette mise en cohérence devrait être soutenue par une communication qui rend transparentes les actions menées par les uns et les autres, pour non seulement impliquer et mobiliser les différentes parties prenantes, mais aussi rassurer tout le monde sur l'utilisation faite des ressources reçues. Dans un pays où les suspicions de corruption sont fréquentes, communiquer et rendre compte accroîtraient la légitimité des décisions prises. 


\subsection{Intégrer la gestion des catastrophes comme relevant des activités courantes de gouvernance du pays}

Jusqu'à présent, la gestion des catastrophes au Cameroun se fait sur un mode épisodique. Le gouvernement tire très peu avantage des expériences passées pour gérer la situation présente. Ainsi, la gestion des crises s'apparente à un éternel recommencement. Pourtant, la récurrence et la fréquence des catastrophes observées ces derniers temps tendent à confirmer que ces phénomènes feront désormais partie du quotidien des populations (GIEC, 2007; Hellendorff, 2012; Amougou, 2018; Bou Nader, 2018).

C'est pourquoi il faut intégrer la gestion des catastrophes comme une activité courante de la gouvernance d'un État, d'une collectivité ou d'une organisation. L'expérience acquise sur le terrain ne suffit donc plus comme étant le mode de développement des connaissances. Désormais, il faut développer une expertise nationale qui s'appuie sur un corpus de connaissances curriculaires. Autrement dit, la gestion des catastrophes doit désormais faire partie des programmes de formation universitaire. Ainsi, le pays serait doté d'une masse critique de spécialistes en gestion de crises qui feraient partie $\mathrm{du}$ corps des fonctionnaires et qui viendraient étoffer les capacités des organisations de la société civile (OSC).

Le nombre d'OSC au Cameroun est estimé à 50000 et $80 \%$ de la population se déclare en être membre (Cazabat, 2015). Gausset (2005) les définit comme l'ensemble des mouvements collectifs, associations, organisations et groupements non gouvernementaux. Dans son plan de développement intitulé Cameroun Vision 2035 (MINEPAT, 2009), le gouvernement accorde un rôle important aux OSC, à savoir offrir des services sociaux pour la lutte contre la pauvreté et la promotion sociale; promouvoir le patrimoine culturel national; être le creuset de la participation et de la mobilisation sociales pour l'intégration des forces productives et pour la démocratisation dans un contexte de décentralisation; et avoir une activité de veille pour la défense des droits de la personne en général, notamment ceux des travailleurs, des femmes, des enfants, des minorités, etc. et pour l'élargissement du processus de démocratisation.
Malheureusement, cette reconnaissance ne s'accompagne pas de moyens. Les OSC camerounaises vivent, pourrait-on dire, dans une situation d'indigence chronique caractérisée par un manque de ressources humaines, financières et matérielles. Dans le cas de la gestion des catastrophes, ces facteurs sont autant de handicaps à l'implication des organisations en temps de crise. La disponibilité de personnes formées en gestion des catastrophes apparait alors comme un premier pas vers la dotation des OSC en ressources humaines qualifiées, le reste devant être pourvu par un organisme pérenne de soutien en temps de crise.

\subsection{Créer un organisme pérenne et spécialisé de soutien aux situations d'urgence}

L'implication des OSC à toutes les phases du processus de gestion des catastrophes est une condition essentielle à la réussite de cet exercice (Shaban, 2001; Linnell, 2013; Miller et Douglass, 2016; Meduri, 2016). Cette implication l'est encore davantage lorsqu'il s'agit de la phase de reconstruction, où l'action gouvernementale doit aller de pair avec les interventions des organismes de première ligne (Daly et collab., 2017).

Dans le cas de la pandémie de COVID-19, la figure 1 ci-dessus montre l'ampleur de ses effets anticipés sur la population. Face à l'imminence de tels effets, le gouvernement camerounais ne peut plus faire l'économie de la prise en charge de la population en période de post-crise. De même, il ne peut plus se contenter de gérer ces périodes au cas par cas, comme il le fait habituellement.

Il est donc temps que le gouvernement camerounais se dote d'une structure permanente de gestion opérationnelle des périodes de post-crise. Une telle structure aura l'avantage de fédérer les expertises en matière de soutien aux populations vulnérables au sein d'un même organisme et de développer des compétences nationales en la matière. L'organisme en question aura une double vocation : collecter les fonds en amont et les distribuer en aval.

En amont, l'organisme aura la charge de rechercher des sources de financement sur une base permanente, soit à travers des campagnes annuelles de financement, soit sur la base d'un impôt (p. ex., une taxation de 1 FCFA par appel téléphonique). Le 
succès d'un tel rôle repose sur deux piliers : la transparence et le caractère apolitique de la structure. La transparence est le gage d'une bonne gestion, tandis que le caractère apolitique garantit que l'organisme ne sert pas un parti politique, mais bien une cause nationale.

En aval, l'organisme en question devrait travailler en étroite collaboration avec les ministères à vocation sociale et accompagner les efforts de soutien aux populations vulnérables des organismes de première ligne. Au Cameroun, ces derniers sont encore en état de végétation, faute de moyens et d'appui. La création d'une structure opérationnelle viendrait combler cette lacune en renforçant les capacités des organismes de première ligne là où ils existent, et en encourageant leur création là où cela s'avère nécessaire.

\section{Conclusion}

Cette étude de cas de gestion de la pandémie de COVID-19 par les autorités camerounaises aux premiers mois de sa survenance a permis de relever les efforts déployés par le gouvernement camerounais pour juguler la crise. Ces efforts se sont déployés sur les plans stratégique, opérationnel et à l'endroit de la population en général. De l'analyse, il ressort que ces efforts auraient pu être plus efficaces s'ils n'avaient pas souffert d'une gestion trop bureaucratique ni de la mise en scène d'une multitude d'acteurs aux rôles parfois mal définis.

$\mathrm{Au}$ regard des crises antérieures survenues au Cameroun, le cas de la pandémie de COVID-19 confirme une gestion au cas par cas des crises dans ce pays. Ce comportement ne favorise pas l'apprentissage organisationnel, de plus en plus nécessaire dans un contexte où la récurrence des crises ne relève plus de la contingence. Ainsi, mettre toutes les énergies dans les phases d'urgence comme c'est le cas actuellement est certes louable, mais insuffisant. C'est pourquoi nous recommandons, pour rendre plus efficace la gestion des crises au Cameroun, d'améliorer la gouvernance en la matière; d'intégrer la gestion des catastrophes comme une activité courante de gouvernance d'un État en l'instituant dans les programmes universitaires; et de créer une structure pérenne de soutien à la gestion des catastrophes.

\section{Limites}

Cet article couvre une période assez courte dans le long effort de gestion de la pandémie de COVID-19; une crise qui s'est installée dans la durée. Il est donc difficile d'en arriver à des conclusions définitives à partir d'une situation en évolution, comme c'est le cas ici ${ }^{4}$. Aussi, faut-il souhaiter que des recherches futures couvrant des périodes plus longues soient menées. On peut penser à des recherches longitudinales non seulement sur la gestion de la pandémie de COVID-19, mais aussi sur la gestion des crises au Cameroun de façon générale. Ces recherches porteraient autant sur la gestion des phases d'urgence que sur celle des phases de reconstruction. Les organismes de première ligne, censés jouer un rôle de premier plan dans la phase de reconstruction, méritent aussi une attention particulière de la part des chercheurs.

\section{NOTES}

1 Le ministère de la Santé publique du Cameroun publie tous les trois jours un bulletin web intitulé Cameroun : rapport de situation COVID-19, qui fait le point sur l'évolution de la pandémie et sur les mesures prises par le gouvernement pour l'endiguer. On apprend ainsi qu’à la fin du mois de juin 2020, le taux de létalité de la COVID-19 au Cameroun est de 2,7 \% pour un taux de guérison de $68 \%$ environ.

2 La polémique ayant entouré la levée de fonds initiée par le parti politique d'opposition Mouvement pour la Renaissance du Cameroun (MRC) pour combattre la pandémie de COVID-19 en est une parfaite illustration. Cette polémique est très vite sortie du champ de la lutte contre la pandémie pour devenir une dispute politicienne.

3 e ministère des Affaires territoriales et de la Décentralisation est désormais scindé en deux : le ministère des Affaires territoriales et le ministère de la Décentralisation et du Développement local.

4 Entre la date de rédaction de la première ébauche de cet article et le moment de sa publication, un certain nombre d'événements sont venus confirmer les faits évoqués. Ainsi, à la suite de l'enquête prescrite par le premier ministre, la Chambre des comptes a relevé dans son rapport (non publié) plusieurs cas avérés de corruption et de distraction de fonds alloués pour la lutte contre la COVID-19. Par la suite, plusieurs ministres ont été entendus par le parquet du Tribunal criminel spécial (TCS). Par ailleurs, un groupe d'étude (task force) pour la riposte contre la COVID-19 a été créé au Secrétariat général de la présidence de la République. Cette task force, de l'avis de plusieurs analystes, crée un bicéphalisme entre le premier ministre et le secrétaire général de la présidence de la République dans le pilotage de la lutte contre la COVID-19. 


\section{RÉFÉRENCES}

Agence française de développement. (2020, 6 mai). Cameroun: 11 millions d'euros d'aide d'ungence pour contenir l'épidémie de COV TD-19. https://www.afd.fr/ fr/actualites/cameroun-11-millions-deuros-daide-durgence-pour-contenir-lepidemie-de-covid-19

Agence France-Presse. (2020a, 25 août). Le bilan mondial de la pandémie de COVID-19 au 25 août. Le Soleil. https://www.lesoleil.com/actualite/monde/le-bilan-mondial-de-la-pandemie-de-COVID-19-au-25-aoutfb4a87374ebad0e52d9c8238d1148063

Agence France-Presse. (2020b, 4 juin). Coronavirus : au Cameroun, la gestion de la pandémie sévèrement critiquée. Le Monde Afrique. https://www.lemonde.fr/afrique/article/2020/06/04/coronavirus-au-cameroun-la-gestion-de-la-pandemie-severementcritiquee_6041729_3212.html

Alexander, D. E. (2000). Confronting catastrophe: New perspectives on natural disasters. Terra Publishing.

Altay, N. et Green, W. G. (2006). OR/MS research in disaster operations management. European Journal of Operational Research, 175(1), 475-493. https://doi.org/10.1016/j.ejor.2005.05.016

Amougou, J. A. (2018). Changements climatiques au Cameroun : manifestations, vulnérabilités, impacts et réponses. Dans KonradAdenauer-Stiftung (dir.), La science, l'économie et la politique du changement climatique : un guide pour les décideurs politiques au Cameroun (p. 5-15). Konrad-Adenauer-Stiftung.

Banque mondiale. (2018). Cameroun - Revue des dépenses publiques : aligner les dépenses publiques aux objectifs de la vision 2035. https://documents1.worldbank.org/curated/en/412641543396425023/pdf/124725-REVISED-FRENCH-Cameroun-revuedepenses-publiques-2018-FINAL.pdf

Banque mondiale. (2020, 8 juin). La pandémie de COV TD-19 plonge l'économie planétaire dans sa pire récession depuis la Seconde Guerre mondiale [communiqué de presse]. https://www.banquemondiale.org/fr/news/press-release/2020/06/08/covid-19-to-plunge-globaleconomy-into-worst-recession-since-world-war-ii

Ben Massou, S. M. (2011, 18-22 mai). Introduction sur la gestion des risques et des catastrophes. Actes de la Working Week 2011 Bridging the gap between cultures de la FIG, Marrakech, Maroc. https://www.fig.net/resources/proceedings/fig_proceedings/fig2011/ papers/ts08b/ts08b_benmassou_4842.pdf

Ben Tchinda Ngoumela, T. (2010). Le système de prévention et de gestion des catastrophes environnementales au Cameroun et le droit international de l'environnement [Mémoire de maitrise, Université de Limoges]. Memoire Online. https://www.memoireonline.com/10/10/4030/m_Le-systeme-de-prevention-et-de-gestion-des-catastrophesenvironnementales-au-Cameroun-et-le-droit-i16.html

Bou Nader, P. (2018). Le changement climatique comme multiplicateur de conflits armés. Les Champs de Mars, $30+$ suppl., $449-456$. https://doi.org/10.3917/lcdm.030.0449

Buissonnière, M. (2012). La nouvelle donne de la santé globale : dynamiques et écueils. International Development Policy/Revue internationale de politique de développement, 3. https://doi.org/10.4000/poldev.953

Burnett, J. J. (1988). A strategic approach to managing crises. Public Relations Review, 24(4), 475-488. https://doi.org/10.1016/S03638111(99)80112-X

Cazabat, C. (2015). Le rôle des organisations de la société civile camerounaises dans la réalisation des Objectifs du Millénaire pour le Développement [Thèse de doctorat, Université Paris-Sorbonne (Paris IV)]. https://www.researchgate.net/publication/291148209_Le_role_des_ organisations_de_la_societe_civile_camerounaises_dans_la_realisation_des_Objectifs_du_Millenaire_pour_le_ Developpement_Universite_Paris-Sorbonne_Paris_IV_Janvier_2015_httpwwwe-sorbonnefrsi

Chandrasekhar, D. (2012). Digging deeper: Participation and non-participation in post-disaster community recovery. Community Development, 43(5), 614-629. https://doi.org/10.1080/15575330.2012.730538

Chang, Y., Wilkinson, S., Brunsdon, D., Seville, E. et Potangaroa, R. (2011). An integrated approach: Managing resources for post-disaster reconstruction. Disasters, 35(4), 739-765. https://doi.org/10.1111/j.1467-7717.2011.01240.x

Chmutina, K. et Von Meding, J. A. (2019). Dilemma of language: "Natural disasters" in academic literature. International Journal Disaster Risk Science, 10, 283-292. https://doi.org/10.1007/s13753-019-00232-2

Clinton, W. J. (2006). Lessons learned from tsunami recovery: Key propositions for building back better. Office of the UN Secretary-General's Special Envoy for Tsunami Recovery. https://www.preventionweb.net/files/2054_VL108301.pdf 
Covington, J. et Simpson, D. M. (2006). An overview of disaster preparedness literature: Building blocks for an applied bay area template. https://www.researchgate.net/publication/242698586_An_Overview_of_Disaster_Preparedness_Literature_ Building_Blocks_for_an_Applied_Bay_Area_Template

CRTV. (2020, 19 mai). Discours spécial du président Paul Biya à la nation le 19 mai 2020 [vidéo]. https://www.crtv.cm/2021/05/ discours-special-du-president-paul-biya-a-la-nation-le-19-mai-2020/

Daly, P., Ninglekhu, S., Hollenbach, P., Duyne Barenstein, J. et Nguyen, D. (2017). Situating local stakeholders within national disaster governance structures: Rebuilding urban neighbourhoods following the 2015 Nepal earthquake. Environment and Urbanization, 29(2), 403-424. https://doi.org/10.1177/0956247817721403

Direction de la protection civile (DPC). (2003). Prévention des risques et gestion des catastrophes au Cameroun. Ministère de l'Administration territoriale et de la Décentralisation, gouvernement du Cameroun. https://www.preventionweb.net/files/889_Cameroonreport.pdf

Dynes, R. R. (1994). Community emergency planning: False assumptions and inappropriate analogies. International Journal of Mass Emergencies and Disasters, 12(2), 141-158. http://udspace.udel.edu/handle/19716/1626

FEMA (2011). National Disaster Recovery Framework: Strengthening Disaster Recovery for the Nation. https:/ /www.fema.gov/pdf/ recoveryframework/ndrf.pdf

Foute, F. (2020a, 22 mai). Samuel Kleda, l'archevêque de Douala, a-t-il trouvé la potion magique contre le coronavirus? Jeune Afrique. https://www.jeuneafrique.com/985864/societe/cameroun-samuel-kleda-un-eveque-au-front-contre-le-COVID-19

Foute, F. (2020b, 6 août). Au Cameroun, des soupçons de malversations autour de la gestion des fonds du Covid-19. Jeune Afrique. https://www.jeuneafrique.com/1025827/politique/au-cameroun-des-soupcons-de-malversations-autour-de-la-gestion-desfonds-du-COVID-19

Fritz, C. (1961). Disaster. Dans R. Merton et R. Nisbet (dir.), Contemporary social problems (p. 651-694). Harcourt.

Gausset, Q. (2005). Organisations communautaires de base et renforcement de la société civile en milieu rural (Adamawa, Cameroun). Civilisations, 52(2), 129-146. https://doi.org/10.4000/civilisations.799

Groupe d'experts intergouvernemental sur l'évolution du climat (GIEC). (2007). Rapport de synthèse : annexe I. Cambridge University Press. https://archive.ipcc.ch/publications_and_data/ar4/syr/fr/annexessannexes-1.html

Halvorson, S. J. et Hamilton, J. P. (2010). In the aftermath of the Qa'yamat: The Kashmir earthquake disaster in northern Pakistan. Disasters, 34(1), 184-204. https://doi.org/10.1111/j.1467-7717.2009.01124.x

Harissi, M. A. et Tourne, I. (2020, 22 juin). Loin de reculer, la pandémie «s'accélère». Le Soleil. https://www.lesoleil.com/ actualite/COVID-19/loin-de-reculer-la-pandemie-saccelere-69e08a7f18c9ed12e8e444de5ee672f7

Hellendorff, B. (2012, 2 octobre). Changement climatique et conflits agro-pastoraux au Sahel [note d'analyse]. Groupe de recherche et d’information sur la paix et la sécurité. https://grip.org/wp-content/uploads/2012/10/NA_2012-10-02_FR_BHELLENDORFF.pdf

Human Right Watch (HRW). (2020, 12 juin). Cameroun : enquêter sur l'utilisation du Fonds de solidarité pour la santé. https://www.hrw.org/fr/news/2020/06/12/cameroun-enqueter-sur-lutilisation-du-fonds-de-solidarite-pour-la-sante\#

Kijewski-Correa, T. et Taflanidis, A. (2012). The Haitian housing dilemma: Can sustainability and hazard resilience be achieved? Bulletin of Earthquake Engineering, 10(3), 765-771. https://doi.org/10.1007/s10518-011-9330-y

Kreps, G. A. (1991). Organizing for emergency management. Dans T. E. Drabek et G. J. Hoetmer (dir.), Emergency management: Principles and practice for local governments (p. 30-54). International City Management Association.

Labadie, J. R. (2008). Auditing of post-disaster recovery and reconstruction activities. Disaster Prevention and Management, 17(5), 575-586. https://doi.org/10.1108/09653560810918612

Le Monde Afrique. (2020, 16 mars). Coronavirus : les pays africains multiplient les mesures bien plus précocement que l'Europe. https://www.lemonde.fr/afrique/article/2020/03/16/covid-19-le-tour-d-afrique-des-mesures_6033295_3212.html

Lewis, J. (2003). Housing construction in earthquake-prone places: Perspectives, priorities and projections for development. The Australian Journal of Emergency Management, 18(2), 35-44. https://www.researchgate.net/publication/266477478_Housing construction_in_earthquake-prone_places_Perspectives_priorities_and_projections_for_development 
Lindblom, C. E. (1959). The science of "muddling through". Public Administration Review, 19(2), 79-88. http://www.jstor.org/ stable/973677

Lindell, M. et Perry, R. (2003). Preparedness for Emergency Response: Guidelines for the Emergency Planning Process. Disasters, 27(4): 336-350

Linnell, M. (2013). Community approaches involving the public in crisis management: A literature review. Risk and Crisis Research Centre, Mittuniversitetet. https://www.researchgate.net/publication/285512375_Community_approaches_involving_the_public_in_ crisis_management_A_literature_review

Lyonnel, C. (2020, 13 mai). COVID-19 au Cameroun : les mesures barrières prises à la légère. La Voix de l'Afrique. https:/ / fr.africanews.com/2020/05/13/covid-19-les-camerounais-deplorent-le-laxisme-des-populations

Lyons, M. (2009). Building back better: The large-scale impact of small-scale approaches to reconstruction. World Development, 37(2), 385-398. http://www.sciencedirect.com/science/article/pii/S0305-750X(08)00147-2

Maher, A. (2006). General rules for dealing with crises (crisis management). Alexandria University House.

Manaouda, M. (2020). Médecine traditionnelle : le ministre de la Santé publique fait le point au parlement. Bulletin de Médecine Traditionnelle, 1, 3-7.

Mbeng Boum, J. (2020). La stratégie de riposte de la région du Nord contre le COVID-19 est prête mais il faut encore matériel de protection et prise en charge. Échos Santé, 157, 8. https://echosante.info/wp-content/uploads/2020/04/Echos-sante157_WEB.pdf

Mbwe Mpoh, M. (2020). Sur le chemin des solutions endogènes. Bulletin de Médecine Traditionnelle, 1, 27-28.

Mbwe Mpoh, M. et Briand, K. J. (2020). La COVID-19 à la rescousse de la médecine traditionnelle? Bulletin de Médecine Traditionnelle, 1, 23.

McEntire, D. A. (2003). Disaster preparedness. International City/County Management Association.

Meduri, Y. (2016). Multi-stakeholder participation in disaster recovery: A case study. Procedia Engineering, 159, 179-185. https://doi.org/10.1016/j.proeng.2016.08.153

Miller, M. et Douglass, M. (2016). Introduction: Decentralising disaster governance in urbanising Asia. Habitat International, 52, 1-4. https://doi.org/10.1016/j.habitatint.2015.08.028

Ministère de l'Administration territoriale et de la Décentralisation (MINATD). (1996). Décret no 96/054 du 12 mars 1996 fixant la composition et les attributions du Conseil national de la protection civile. Gouvernement du Cameroun. http://bibliotheque.pssfp.net/livres/receuil_des_textes_juridiques_minatd.pdf

Ministère de l'Administration territoriale et de la Décentralisation (MINATD). (1998). Décret no 98/031 du 09 mars 1998 portant organisation des plans d'urgence et des secours en cas de catastrophe ou de risque majeur. Gouvernement du Cameroun. http://bibliotheque.pssfp.net/livres/receuil_des_textes_juridiques_minatd.pdf

Ministère de l'Économie, de la Planification et de l'Aménagement du territoire (MINEPAT). (2009). Cameroun Vision 2035 : document de travail. Gouvernement du Cameroun. http://extwprlegs1.fao.org/docs/pdf/cmr145894.pdf

Ministère de la Santé publique. (2020). Plan de préparation et de réponse an COVID-19 au Cameroun. Gouvernement du Cameroun. https://reliefweb.int/sites/reliefweb.int/files/resources/plan_de_preparation_coronavirus_cmr._version_du_10_mars_2019.pdf

Ministère des Finances. (2020a). Décret n 2020/3221 du 22 juillet 2020 fixant la répartition de la dotation du Fonds spécial de solidarité nationale pour la lutte contre le coronavirus et ses répercussions économiques et sociales. Gouvernement du Cameroun. http:/ /www.minfi.gov.cm/ wp-content/uploads/2020/07/decret_repartition_covid19_du_22_juil_2020_fr.pdf

Ministère des Finances. (2020b). Ordonnance no 2020/001 du 03 juin 2020 modifiant et complétant certaines dispositions de la loi no 2019/023 du 24 décembre 2019 portant loi de finances de la République du Cameroun pour l'exercice 2020. Gouvernement du Cameroun. https://www.prc.cm/fr/multimedia/documents/8326-ordonnance-n-2020-001-du-03-06-2020-lite

Moe, T. L. et Pathranarakul, P. (2006). An integrated approach to natural disaster management: Public project management and its critical success factors. Disaster Prevention and Management, 15(3), 396-413. https://doi.org/10.1108/09653560610669882

National Academy of Sciences (NAS). (2011). Building community resilience through private-public collaboration. National Academies Press. https://research.fit.edu/media/site-specific/researchfitedu/coast-climate-adaptation-library/united-states/national/us---othernational-reports/NRC.-2011.-Private--Public-Collaboration-for-Disaster-Resilience.pdf 
Newport, J. et Jawahar, G. (2003). Community participation and public awareness in disaster mitigation. Disaster Prevention and Management, 12(1), 33-36. https://doi.org/10.1108/09653560310463838

Ntchapda, A. (2020). Samuel Kleda déclare qu’il a «vaincu le coronavirus», prudence! Allo Docteurs Africa. https://www.allodocteurs.africa/samuel-kleda-declare-qu-il-a-vaincu-le-coronavirus-prudence-4265.html

Nudell, M. et Antokol, N. (1988). The handbook for effective emergency and crisis management. Lexington Books.

OECD (2003), Emerging Risks in the 21st Century, An Agenda for Action, OECD Publishing.

ONUSIDA. (2020). Gérer l'épidémie de COVID-19 an Cameroun. https://www.unaids.org/fr/resources/presscentre/ featurestories/2020/may/20200511_COVID19-cameroon

Organisation mondiale de la santé (OMS). (2018). État de la santé dans la Région africaine de l'OMS : analyse de la situation, des services et des systèmes de santé dans le contexte des objectifs de développement durable. Bureau régional de l'Afrique. https:/ apps.who.int/iris/ handle/10665/275278

Organisation mondiale de la santé (OMS). (2020, 31 janvier). Surveillance mondiale de l'infection bumaine par le nouveau coronavirus (2019-nCoV) : orientations provisoires. https://apps.who.int/iris/handle/10665/330858

Pahai, O. et Toussaint, V. (2020). Cameroun-Covid-19 : Atanga Nji préside le départ du don de Paul Biya dans les 10 régions. Blasting News. https:// fr.blastingnews.com/international/2020/04/cameroun-COVID-19-atanga-nji-preside-le-depart-du-don-de-paulbiya-dans-les-10-regions-003127515.html

Pielke, R. A. Sr. et Pielke, R. A. Jr. (1997). Hurricanes: Their nature and impact on society. Wiley.

Premier ministre du Cameroun. (2003). Arrêté no 037/PM du 19 mars 2003 portant création, organisation et fonctionnement d'un observatoire national des risques. Gouvernement du Cameroun. https://droitcamerounais.info/en/files/128.03.03-Arrete-du-19-mars-2003_ Observatoire-national-des-risques.pdf

Programme des Nations unies pour le développement (PNUD). (2020). Effets socioéconomiques potentiels du Covid-19 au Cameroun : une évaluation sommaire. https://www.undp.org/content/dam/rba/docs/COVID-19-CO-Response/Socio-Economic-ImpactCOVID-19-Cameroon-UNDP-Cameroon-March-2020.pdf

Quarantelli, E. L. (dir.). (1998). What is a disaster? Perspectives on the question. Routledge.

Quenet, G. (2000). La catastrophe, un objet historique? Hypothèses, 3(1), 11-20. https://doi.org/10.3917/hyp.991.0011

Rodriguez, H., Quarantelli, E. L. et Dynes, R. R. (2007). Handbook of disaster research. Springer.

Sellnow, T. et Sellnow, D. (2010). The instructional dynamic of risk and crisis communication: Distinguishing instructional messages from dialogue. Review of Communication, 10(2), 112-126. https://doi.org/10.1080/15358590903402200

Shaban A. (2001, 29-31 janvier). Experiences of education and community involvement. Actes de l'International Workshop on earthquake safer world in the 21 st century de l'UNCRD, Kobe, Japon.

Shafique, K. et Warren, C. M. J. (2016). Stakeholders and their significance in post natural disaster reconstruction projects: A systematic review of the literature. Asian Social Science, 12(10). https://dx.doi.org/10.5539/ass.v12n10p1

Shaw, R. (2006). Indian Ocean tsunami and aftermath: Need for environment-disaster synergy in the reconstruction process. Disaster Prevention and Management, 15(1), 5-20. https://doi.org/10.1108/09653560610654202

Sutton, J. et Tierney, K. (2006). Disaster preparedness: Concepts, guidance, and research. Natural Hazards Center Institute of Behavioral Science, University of Colorado Boulder.

Tchingankong Yanou, M. (2014). La gestion par le haut des catastrophes au Cameroun : une expression de l'apprentissage étatique des politiques publiques. Polis, 19(1-2), 101-127. https://www.researchgate.net/publication/331314789_

La_gestion_par_le_haut_des_catastrophes_au_Cameroun_Une_expression_de_l\%27apprentissage_etatique_des_politiques_ publiques

Turner, J. R. et Muller, R. (2004). Communication and co-operation on projects between the project owner as principal and the project manager as agent. European Management Journal, 22(3), 327-336. https://doi.org/10.1016/j.emj.2004.04.010

United Nations Office for Disaster Risk Reduction (UNISDR). (2005). Cadre d'action de Hyogo pour 2005-2015: pour des nations et des collectivités résilientes face aux catastrophes. https://www.unisdr.org/2005/wcdr/intergover/official-doc/L-docs/Hyogo-frameworkfor-action-french.pdf 
United Nations Office for Disaster Risk Reduction (UNISDR). (2009). Terminologie pour la prévention des risques de catastrophe. https://www.preventionweb.net/files/7817_UNISDRTerminologyFrench.pdf

United Nations Office for Disaster Risk Reduction (UNISDR). (2015). Cadre d'action de Sendai pour la réduction des risques de catastrophe 2015-2030. https://www.unisdr.org/files/43291_frenchsendaiframeworkfordisasterris.pdf

Valensisi, G. (2020). COVID-19 et pauvreté mondiale : une évaluation préliminaire. International Development Policy/Revue internationale de politique de développement, 12(2). https://doi.org/10.4000/poldev.3573

Wybo, J.-L. (2010). L'évaluation de la vulnérabilité à la crise : le cas des préfectures en France. Télescope, 16(2), 173-193. https://telescope.enap.ca/Telescope/docs/Index/Vol_16_no_2/Telv16n2_wybo.pdf

Xie, Z. (1994, 1-4 novembre). Seismic damage and reconstruction of Tangsha city. Actes de l'Aichi/Nagoya International Conference de l'UNCRD, Nagoya, Japon.

Ye, Y. et Okada, N. (2002). Integrated relief and reconstruction management following a natural disaster. Actes du Second Annual IIASA-DPRI Meeting, Integrated Disaster Risk Management: Megacity Vulnerability and Resilience de l'IBWPAN, Laxenbourg, Autriche. 